\title{
Molecular epidemiology and survival analysis of nosocomial meningitis induced by multi-drug resistance Enterobacteriaceae
}

\author{
Guanghui Zheng ${ }^{1}$, Yanfei Cao ${ }^{1}$, Lingye Qian ${ }^{1}$, Yumeng $\mathrm{Cai}^{1}$, Hong $\mathrm{Lv}^{1}$, Lina Zhang ${ }^{1}$, and \\ Guojun Zhang ${ }^{1}$
}

${ }^{1}$ Affiliation not available

November 30, 2020

\begin{abstract}
Objectives: To evaluate the molecular epidemiology and mortality risk factors of nosocomial meningitis (NM) induced by multidrug resistance Enterobacteriaceae (MDRE) in China. Methods: We performed a multi-center study of MDRE NM patients in 2 neurosurgical centers in China from 2014 to 2019. Molecular and phenotype microbiology epidemiology of each MDRE were reviewed and tested, and 21 clinical variables on mortality risk factors were extracted and evaluated by multivariate Cox analysis for NM. Results: In total, 90 MDRE NM patients were included in this study. Klebsiella (K.) pneumoniae occupied the highest proportion $(51.11 \%, 46 / 90), 44(44.44 \%)$ were meropenem-resistant, ceftriaxone resistance in target MDRE was relatively high $(92.22 \%, 83 / 90)$, blaKPC $(67.50 \%, 27 / 40)$ was the predominant carbapenem resistance gene, and blaCTX-M1 , blaTEM and blaCTM-M-9 were the three most popular extended spectrum $\beta$-lactamases (ESBLs) producing genes of the MDRE. Multivariate Cox analysis showed that external ventricular drainage (EVD, odds ratio (OR) 2.524, 95\% confidence interval (CI) 1.101-5.787, P = 0.029) and Glasgow Coma Scale (GCS) [?]8 (OR 4.033, 95\% CI 1.526-10.645, P =0.005) were mortality risk factors of MDRE NM. Conclusions: NM caused by MDRE is an important sign of the failure of neurosurgery, and MDRE has multiple drug resistance genotypes, and EVD and GCS[?]8 are independent mortality risk factors of MDRE NM, which deserves the attention of microbiologist and neurosurgical clinicians.
\end{abstract}

\section{Introduction}

Nosocomial meningitis (NM) is a serious neurosurgical complication, which severely affects the survival of the patients and success rate of neurosurgery. Patients with NM always suffered relatively large-scale surgery, and the body's immunity was lower. According to reports in the literature, compared with non-nosocomial meningitis patients, the mortality of NM patients is up to $15-45 \%{ }^{[1]}$

Increasing antibiotic resistance in Enterobacteriaceae is a growing public health crisis that makes many medical care-associated infections hard to treat with current antibiotics ${ }^{[2,3]}$. Multi-drug resistant Enterobacteriaceae (MDRE) is defined as an Enterobacteriaceae resistant to at least three types of antibiotics ${ }^{[4]}$, including $\beta$-lactams, aminoglycosides, macrolides, quinolones antibiotics and so on. Because of their multiple drug resistance properties, clinicians are always limited in their choice of antibiotics. NM caused by MDRE usually leads to serious consequences of the neurosurgical patients. Due to the complexity of neurosurgery and the existence of a blood-brain barrier, the mortality rate of MDR bacterial meningitis is always higher than that of wild-type strains ${ }^{[5]}$.

This study focus on the molecular epidemiology, clinical features of the largest series of MDRE-NM reported to date, with the aim of evaluating the clinical outcome of NM caused by MDRE, and examining the risk factors for mortality in MDRE-NM. To my best of knowledge, this is the first survival analysis on MDRE-NM.

\section{Materials and Methods}




\section{Study design}

This study was performed at two neurological centers in northern China between Jan 2014 to Dec 2019, including Beijing Tiantan Hospital and Capital Medical University, and Daqing Oilfield General Hospital. All of the patients eligible were enrolled from neurosurgical wards of the two centers listed above. The molecular characteristic test of this study was approved by the ethical committee of Beijing Tiantan Hospital and Capital Medical University (Approved Number: KY-2019-095-03).

\section{Definition of the MDRE-NM}

MDRE: Enterobacteriaceae resistant to three or more types of antibiotics, including $\beta$-lactams (meropenem and ceftriaxone), aminoglycosides (amikacin), quinolones (levofloxacin), tetracyclines, polymyxins B, sulfamethoxazole and trimethoprim (SMZ-TMP), and chloramphenicol.

Patients were eligible if they were older than 18 years and survived at least 7 days after neurosurgery. Neurosurgical operations included craniotomy and trans-sphenoidal and spinal surgeries. Patients without neurosurgery, anti-infective operations not done in their hospital, and incomplete medical records were excluded from our study. The patients were followed for the development of NM during the first 30 postoperative days. The inclusion criteria are: 1) MDRE cultured from the patients' CSF;

2 ) at least one of the symptoms or signs followed with no other cause: headache, fever( $>38)$, neurological abnormalities(stiff neck, meningeal signs, cranial nerve signs or irritability), despite appropriate antibiotic therapy; 3) at least one of the abnormal clinical laboratory tests: a) elevated CSF leucocyte count, increased CSF protein level, and/or decreased CSF glucose concentration; b) Enterobacteriaceae cultured from blood. The flow chart and exclusion criteria are shown in Figure-1.

\section{Microbiology}

All of the cerebrospinal fluid (CSF) specimens were collected from the patients of two neurosurgical centers. The MDRE isolated from the CSF underwent culture, bacterial identification, antimicrobial susceptibility tests (AST), and resistance gene screening. CSF (1-3 ml) of the patients was obtained either by lumbar puncture or from CSF devices, and were inoculated into bacterial culture bottles for automatic culture. When the culture system alarm for positive, $20 \mu \mathrm{l}$ of bacterial culture broths were sub-cultured on $5 \%$ Columbia sheep blood agar plates (bioMerieux, Marcy l'Etoile, France) and MacConkey (bioMerieux, Marcy l'Etoile, France) and incubated aerobically at $37^{\circ} \mathrm{C}$ for $18-24$ hours. All suspected bacteria with growth characteristics of Enterobacteriaceae were subjected to standard microbial identification procedures.

The systems employed for microbial identification are VITEK-2 Compact (bioMerieux, Marcy l'Etoile, France), and VITEK MS (bioMerieux, Marcy l'Etoile, France) systems. AST of Enterobacteriaceae was performed by the Kirby-Bauer method and broth microdilution method (MIC) and classified as sensitive, intermediate, and resistant according to the Clinical and Laboratory Standards Institute (CLSI) ${ }^{[6]}$. Resistance gene detection of MDRE were carried out by the second-generation micro/nanofluidic chip platform-B (MNCP-II-B) based on the loop-mediated isothermal amplification method (LAMP) ${ }^{[7]}$. The extraction of the nucleic acid and test procedure was done according to the MNCP-II-B manufacturer's instructions ${ }^{[8]}$.

\section{Patients' characteristics and risk factors}

All of the NM patients' qualified daily medical records were extracting from the database of the neurosurgery, clinical infectious diseases, and microbiology departments in the two centers described above. From that, 21 characteristics were selected as the mortality risk factors of the NM patients, including: Age, Male\%, Hypertension, Diabetes mellitus, Fever (b.t $>38$ ), lumbar drainage (LD), external ventricular drainage (EVD), long-time surgery duration(>180 min), CSF leakage, reoperation, assisted mechanical ventilation(AMV), length of hospital stay (LOS), time of cure of infection (days), surgical wound classification, craniotomy, Glasgow Coma Scale (GCS) ([?]8), Positive culture time, ICU admission, Malignancy, Bacterimia and Hospital acquired pneumonia(HAP).

Statistics Analysis 
Whonet 5.5 and SPSS (IBM, New York, US version 22) were performed as the statistical analysis. Categorical data were expressed as numbers and percentages, whereas continuous data were expressed as median +- standard deviations (SDs) or median with interquartile range (IQR). In univariate analysis, normally distributed quantitative variables were analyzed with either Student's t-test or one-way analysis of variance test as appropriate. Abnormally distributed quantitative variables were processed using Mann-Whitney $\mathrm{U}$ test, and categorical data were assessed by Pearson's chi-squared $(\chi 2)$ test. Univariate analysis was employed to calculate the $\mathrm{P}$ values for all variables, and $\mathrm{K}-\mathrm{M}$ analysis was performed in the univariate variable with $\mathrm{p}<0.05$. A multivariate Cox regression algorithm was performed to predict the MDRE-NM mortality, and the results were expressed as $\mathrm{P}$ value, sub-distribution hazard ratio (HR), and their $95 \%$ confidence intervals (C.I.). Any variables with $\mathrm{P}<0.1$ in the Kaplan-Meier (K-M) survival analysis were carried forward into the multivariate Cox regression algorithm to analyze the independent mortality risk factor for MDRE-NM. Significance was defined as a $\mathrm{P}<0.05$, and calibration was assessed using the Hosmer-Lemeshow (H-L) test for goodness-of-fit

\section{Results}

During the study period, 45,771 neurosurgery patients and 3,570 PNM patients were included in the two centers described above. The infection rate was $7.8 \%(3,570 / 45,771)$. Among them, 242 cases ofEnterobacteriaceae contained 41.73\%(101/242) of patients with MDRE-NM were recorded, Of them, 2 patients younger than 18 years old, 3 patients hospitalized less than 7 days or without antimicrobial treatment, 2 patients that died within 7 days and 4 patients with incomplete medical records were (11) excluded from this study. Of the remaining 90 cases, 79 patients were from Beijing Tiantan Hospital and Capital Medical University (55 survival, 24 Non-survival), and 11 were from Daqing Oilfield General Hospital (7 survival, 4 Non-survival). The flow sheet of this study is shown in Figure-1, and distribution of the MDRE-NM patients is shown in Figure-2.

\section{Microbiology}

Isolate distributions of Enterobacteriaceae and MDRE are shown in Table-1. From that, Klebsiella pneumoniae occupied the highest MDRE proportion (51.11\%, 46/90), followed by Escherichia coli $(26.67 \%, 24 / 90)$, Klebsiella aerogenes $(8.89 \%, 8 / 90)$, and Enterobacter cloacae $(5.56 \%, 5 / 90)$.

\section{$A S T$ and Genotyping of MDRE}

Table-2 shows the AST and genotyping of MDRE in 90 NM patients. Among them, 44 (44.44\%) were meropenem-resistant, and ceftriaxone resistance in target MDRE was relatively high $(92.22 \%, 83 / 90)$, whereas 32.22\%(29/90) resistance was found in amikacin. No polymyxin B-resistant MDRE was found in this study, and in addition, MDRE has a relatively lower resistance to SMZ-TMP $(18.89 \%, 17 / 90)$. The resistance rates of MDRE to tetracycline, levofloxacin, and chloramphenicol were relatively similar, being $66.57 \%$ (60/90), $74.44 \%$ (67/90), and $63.33 \%$ (57/90), respectively.

Meropenem-resistant MDRE contained at least one carbapenem related gene and the bla $a_{K P}(67.50 \%, 27 / 40)$ was the predominant gene. In addition, extended spectrum $\beta$-lactamases (ESBLs) producing genes+Ompk35 $(65.00 \%, 26 / 40)$ is also a more popular gene form of resistance, and bla OXA-66 accounts for $27.5 \%(11 / 40)$. In total, bla $a_{S H V}, b l a_{T E M}$, and bla $a_{C T X-M-9}$ are the 3 most popular ESBLs producing genes of the MDRE. The whole genotype distributions are shown in Table-2.

\section{Comparative Analysis between Survival and Non-Survival patients}

Characteristics of patients stratified by survival and non-survivalpatients are presented in Table-3. The main demographic and clinical characteristic data of both groups are shown in Table-3. Patients with MDRE-NM had a median age of 49 years (IQR, 31-59), and $64.44 \%$ (58/90) of patients were male. Hypertension was found in $22.22 \%$ (20/90) of patients, diabetes mellitus in 5.56\% (5/90), 43.33\% (39/90) had bacteremia and HAP comorbidity in $40.00 \%$ (36/90). Among the 90 cases, $64.44 \%$ (58/90) of patients had fever, $51.11 \%$ (46/90) had EVD, 41.11\% (37/90) had LD and 21.11\% (19/90) had CSF leakage. More than half (57.78\%, 
$52 / 90$ ) of patients experienced long surgery duration (>180 $\mathrm{min}$ ), 27.78\% (25/90) of patients underwent reoperation, and $42.22 \%(38 / 90)$ of patients were placed on a ventilator. The median LOS of the MDRENM patients was 27 (IQR, 20-42) days, and the median time of CSF-positive culture was 7 (IQR, 3-13) days. Time of cure of infection was 12 (IQR, 7-18) days. Incidence of craniotomy surgery and malignancy were $68.89 \%$ (62/90) and $57.78 \%$ (52/90), and the ICU admission rate was $47.78 \%$ (43/90). Clean surgical wound operation made up $62.22 \%$ (56/90) of cases, and the remaining patients' experienced a clean-contaminated surgical wound operation.

\section{Outcome and survival analysis}

Overall 30-day mortality was $31.11 \%$ (28/90). Univariate analysis showed that EVD, AMV, GCS[?]8, ICU admission, bacteremia and HAP had significant differences between the survival and non-survival groups, and K-M survival analysis showed that EVD and GCS[?]8 had significant differences between the two groups. The results are shown in Figure-3. Multivariate Cox survival analysis showed that EVD (HR 2.524, 95\% C.I. $1.101-5.787, P=0.029$ ) and GCS[?]8 (HR 4.033, 95\% C.I. $1.526-10.645, P=0.005$ ) were mortality risk factors of MDRE-NM.

\section{Discussion}

The emergence and spread of MDR pathogens, especiallyEnterobacteriaceae, is an important nosocomial health concern, making better evaluation of the molecular characterization and mortality risk factors of these strains vitally important. In this case-control study, molecular characteristic and mortality risk factors of MDRE-NM were evaluated. The results of this article revealed a high rate $(41.73 \%, 101 / 242)$ of MDRE proportion of the EnterobacteriaceaeNM in neurosurgical patients, among which K. pneumoniae $(51.11 \%, 46 / 90)$ was the most in prevalent Enterobacteriaceae causing MDRE-NM. In AST, third-generation cephalosporin (ceftriaxone) and carbapenem (meropenem) have a high resistance rate of $92.22 \%$ and $44.44 \%$, respectively. MDRE has specific resistance genotypes for various antibiotics, among which $b l a_{K P C}(67.50 \%, 27 / 40)$ and $b l a_{C T X-M-1}(72.29 \%, 60 / 83)$ have the highest ratios. Multivariate Cox survival analysis showed that EVD and GCS[?] 8 are independent mortality risk factors of MDRE-NM.

Enterobacteriaceae is one of the most severe pathogenic bacterium that causes nosocomial infections. Due to the limited choice of antibiotics, infections caused by MDRE can lead to more serious outcomes. In recent years, the increasing number of MDRE infections has brought great difficulties to clinical treatment. In the present study, the proportion of $K$. pneumoniae was as high as $51.1 \%$, which exceeded the proportion of Enterobacteriaceae reported in the literature ${ }^{[9]}$, indicating that MDR-K. pneumoniae caused more neurosurgical meningitis. K. pneumoniaenot only possess high drug resistance, but also has high virulence ${ }^{[10]}$ and high invasiveness property ${ }^{[11]}$, and its infection mortality rate is also higher than other types of Enterobacteriaceae [12]. E. coli and K. aerogenes also account for a certain proportion of infections. The three kinds of Enterobacteriaceae account for $86.67 \%$ of all MDREs and are the most important pathogenic bacteria.

The definition of MDR is one bacterium resistant to at least three types of antibiotics. In this study, 8 kinds of antibiotics, including meropenem, ceftriaxone, amikacin, levofloxacin, SMZ-TMP,tetracycline, chloramphenicol and polymyxin B, were classified to screen MDRE. Target MDRE have high resistance rates to meropenem (44.44\%) and ceftriaxone (92.22\%); meanwhile, resistance to tetracycline, levofloxacin, and chloramphenicol is relatively high. Nevertheless, for amikacin, SMZ-TMP, and polymyxin B, the drug resistance rate is low. All MDRE enrolled are especially sensitive to polymixin B, which is the last line of defense against MDR Gram-negative bacteria. There is a report that polymyxin B can be injected intrathecally in patients with severe multi-resistant bacterial infection, which may be a better alternative for MDRE-NM ${ }^{[13]}$. However, polymyxin B possesses several adverse reactions, such as nephrotoxicity ${ }^{[14]}$, neuromuscular blockages ${ }^{[15]}$, and respiratory depression ${ }^{[16]}$, so the application is limited to NM patients.

Antibiotic resistance genes are closely related to the choice of therapeutic drugs. For example, ceftazidime/avicarbatan has better activity against A and D-type carbapenemase-producing bacteria, but it is ineffective against B-type (such as, bla $l a_{V M}$ and $b l a_{I M P}$ ) carbapenemase-producing bacteria ${ }^{[17]}$. The distribution of carbapenemase-producing Enterobacteriaceae genes always varies; for example, the most prominent 
genotype in Europe is D-typebla $a_{X A-48}{ }^{[18]}$, the most prominent strain in India is $b l a_{N D M-1}{ }^{[19]}$, and the most prominent strain in China is $b l a_{K P C}{ }^{[10,20]}$ respectively. In this study, the majority of MDREs possess more than one type of carbapenem-resistant genes; nevertheless, $b l a_{K P C}$ occupied the greatest proportion of the carbapenemase-producing mechanism. Previous studies have reported that bacteria that express loss of membrane porin protein and modifications in permeability caused by efflux pump systems can significantly affect their resistance to carbapenem ${ }^{[21,22]}$. Our results confirm that Enterobacteriaceae express ESBL producing gene+Ompk35 occupied a huge proportion, however, since Ompk35 is not a routine clinical laboratory test, $\mathrm{ESBL}+$ Ompk35, as a form of carbapenem resistance mechanism, also deserves the neurosurgical attention. Studies have confirmed that the genotype of the MDRE predominance varied in regions and locations and to a great extent determined the resistance of the MDRE in the locality. For example, an African study shows that, $b l a_{S H V}(36.4 \%)$ was the predominant gene, followed by $b l a_{T E M}(31.4 \%)$ and $b l a_{C T X-M}(27.3 \%)^{[23]}$. A study from The Netherlands, however, reported $b a_{C T X-M-1}$ as the predominant gene, followed by $b l a_{C T X-M-15}$ [24]. In another European longitudinal analysis of ESBL carriage ofEnterobacteriaceae, bla $C T X-M-15$ was the dominant ESBL in all countries except Greece, wherebla $a_{S H V}$ was more common ${ }^{[25]}$. Screening for the third-generation cephalosporin-related resistance genes in this study revealed that the three highest proportion genotypes ( $b l a_{T E M}, b l a_{C T X-M-9}$, and $b l a_{S H V}$ ) all belong to ESBL-producing resistance genes. Probably because we embedded relatively few genes related to the production of AmpC enzyme, fewer AmpC-related genes were not detected. For amikacin, tetracycline, and levofloxacin, we found that although the MDRE showed resistance, we did not screen out the corresponding resistance genes. The reason may be that our selection of resistance genes is insufficient, and the testing of resistance genes is limited.

Knowledge of the mortality risk factors will help in early treatment of patients suffering from the MDRE$\mathrm{NM}^{[26]}$. This also is a significant step in the prevention of the spread of resistantEnterobacteriaceae among hospitalized neurosurgical patients, and control of the hospital spread will definitely have a role in maintain the health and safety of them. The prognosis of NM usually has poor outcomes, and prior studies reported that the mortality of NM in patients with neurosurgery range between 20 and $78 \%$ [27]. Previous studies have identified numerous risk factors of meningitis, including CSF leakage ${ }^{[28]}$, coma $^{[29]}$, surgical intervention ${ }^{[30]}$, impaired consciousness ${ }^{[31]}$, long surgery duration, AMV, non-sterile technique during the surgery, and delayed removal of the catheter even when clinically indicated ${ }^{[32]}$. In our study, the overall 30-day MDRE-NM mortality was $31.11 \%$. EVD and GCS([?]8) were associated with poor prognosis of MDRE-NM. EVD is common after brain surgery; however, the incidence of EVD caused infection is still relatively high and can increase morbidity and mortality. Surveys such as that conducted by $\mathrm{Lu}^{[33]}$ have shown that the incidence of EVD-related infection may reach $22 \%$ in high-risk patients ${ }^{[34]}$. EVD increases the NM patients' morbidity and mortality as well as prolonging LOS, increasing hospitalization costs, and even causing multiple surgeries $^{[35,36]}$. GCS provides an objective and reliable way of recording the conscious state of a patient. Generally, comas can be classified by GCS: severe, with GCS[?]8, moderate, GCS 9-12, and minor, GCS $>13^{[37]}$. Low GCS was a mortality risk factor and may have been predictive of the poor prognosis of MDRENM in our study. It makes sense that low GCS means severity of the NM and coma, which easily leads the poor outcome.

There are several limitations to our study. First, this is a retrospective study to evaluate the mortality risk factors. This implies that the results of the study may not be representative in comparison with the perspective studies. Second, the relatively small number of patients limited our epidemiologically analysis. Third, numbers of drug resistance genes were relatively small, and many types of the genes were not included. This may require further multicenter studies for mortality risk factor screening, and more resistance gene evaluation for the molecular epidemiology of the MDRE-NM.

\section{Conclusion}

In total, to the best of our knowledge, this is the first study to conduct a two-center molecular and casecontrol study to evaluate the mortality risk factors of MDRE-NM. From our study, MDRE has multiple drug resistance genotypes, and EVD and GCS[?]8 are independent mortality risk factors of MDRE-NM, which deserves the attention of microbiologist and neurosurgical clinicians. 


\section{Acknowledgment}

I want to take this chance to thanks to Yijun Shi, Yan Zhang, they give me many academic and precious suggestions at this section and helps me to correct my paper.

\section{Funding}

This study was supported by Beijing Municipal Administration of Hospitals [grant number: QML20180502 ].

\section{Ethical approval}

This study was approved by the ethical committee of Beijing Tiantan Hospital and Capital Medical University (Approved Number: KY-2019-095-03).

\section{Potential conflicts of interest}

All authors report no conflicts of interest relevant to this article

Author contributions: Guanghui. Zheng, Yanfei Cao: Design; Data collection; Data analysis, interpretation; Lingye Qian: Statistics; Yumeng Cai, Drafting article; Hong Lv: MDR-genotypes test;

Lina Zhang and Guojun Zhang: Conduct the whole study

\section{References}

[1] Pintado V, Pazos R, Jiménez-Mejías ME, et al. Staphylococcus aureus meningitis in adults: A comparative cohort study of infections caused by meticillin-resistant and meticillin-susceptible strains. J Hosp Infect. 2019. 102(1): 108-115.

[2] Liu J, Zhang L, Pan J, et al. Risk Factors and Molecular Epidemiology of Complicated Intra-Abdominal Infections With Carbapenem-Resistant Enterobacteriaceae: A Multicenter Study in China. J Infect Dis. 2020. 221(Supplement_2): S156-S163.

[3] Bielli A, Piazza A, Cento V, et al. In vivo acquisition and risk of inter-species spread of blaKPC-3-plasmid from Klebsiella pneumoniae to Serratia marcescens in the lower respiratory tract. J Med Microbiol. 2020. 69(1): 82-86.

[4] Teklu DS, Negeri AA, Legese MH, Bedada TL, Woldemariam HK, Tullu KD. Extended-spectrum betalactamase production and multi-drug resistance among Enterobacteriaceae isolated in Addis Ababa, Ethiopia. Antimicrob Resist Infect Control. 2019. 8: 39.

[5] Liang W, Yuan-Run Z, Min Y. Clinical Presentations and Outcomes of Post-Operative Central Nervous System Infection Caused by Multi-Drug-Resistant/Extensively Drug-Resistant Acinetobacter baumannii: A Retrospective Study. Surg Infect (Larchmt). 2019. 20(6): 460-464.

[6] Weinstein MP, Lewis JS 2nd. The Clinical and Laboratory Standards Institute Subcommittee on Antimicrobial Susceptibility Testing: Background, Organization, Functions, and Processes. J Clin Microbiol. 2020. $58(3)$.

[7] Zheng G, Zhang Y, Zhang L, et al. Evaluation of a micro/nanofluidic chip platform for diagnosis of central nervous system infections: a multi-center prospective study. Sci Rep. 2020. 10(1): 1568.

[8] Zhang G, Zheng G, Zhang Y, Ma R, Kang X. Evaluation of a micro/nanofluidic chip platform for the high-throughput detection of bacteria and their antibiotic resistance genes in post-neurosurgical meningitis. Int J Infect Dis. 2018. 70: 115-120.

[9] Balkhair A, Al-Muharrmi Z, Al'Adawi B, et al. Prevalence and 30-day all-cause mortality of carbapenemand colistin-resistant bacteraemia caused by Acinetobacter baumannii, Pseudomonas aeruginosa, and Klebsiella pneumoniae: Description of a decade-long trend. Int J Infect Dis. 2019. 85: 10-15. 
[10] Xu M, Fu Y, Fang Y, et al. High prevalence of KPC-2-producing hypervirulent Klebsiella pneumoniae causing meningitis in Eastern China. Infect Drug Resist. 2019. 12: 641-653.

[11] Juan CH, Chuang C, Chen CH, Li L, Lin YT. Clinical characteristics, antimicrobial resistance and capsular types of community-acquired, healthcare-associated, and nosocomial Klebsiella pneumoniae bacteremia. Antimicrob Resist Infect Control. 2019. 8: 1.

[12] Park JW, Lee H, Park SY, Kim TH. Epidemiological, clinical, and microbiological characteristics of carbapenemase-producing Enterobacteriaceae bloodstream infection in the Republic of Korea. Antimicrob Resist Infect Control. 2019. 8: 48.

[13] Abad-Restrepo J, Díaz-Díaz A, Osorio-Cadavid N. [Post-surgical ventriculitis due to extensively resistant Pseudomonas aeruginosa treated with intrathecal colistin. Pediatric case report and literature review]. Rev Chilena Infectol. 2018. 35(3): 321-325.

[14] Zavascki AP, Nation RL. Nephrotoxicity of Polymyxins: Is There Any Difference between Colistimethate and Polymyxin B. Antimicrob Agents Chemother. 2017. 61(3).

[15] Myint T, Evans ME, Burgess DR, Greenberg RN. Respiratory Muscle Paralysis Associated With Colistin, Polymyxin B, and Muscle Relaxants Drugs: A Case Report. J Investig Med High Impact Case Rep. 2016. 4(1): 2324709616638362.

[16] Hussein MH, Daoud GA, Kakita H, et al. Effect of polymyxin B-immobilized fiber hemoperfusion on respiratory impairment, hepatocellular dysfunction, and leucopenia in a neonatal sepsis model. Pediatr Surg Int. 2010. 26(2): 187-93.

[17] Zhanel GG, Lawson CD, Adam H, et al. Ceftazidime-avibactam: a novel cephalosporin/ $\beta$-lactamase inhibitor combination. Drugs. 2013. 73(2): 159-77.

[18] van Duin D, Doi Y. The global epidemiology of carbapenemase-producing Enterobacteriaceae. Virulence. 2017. 8(4): 460-469.

[19] Rahman M, Mukhopadhyay C, Rai RP, et al. Novel variant NDM-11 and other NDM-1 variants in multidrug-resistant Escherichia coli from South India. J Glob Antimicrob Resist. 2018. 14: 154-157.

[20] Chi X, Hu G, Xu H, et al. Genomic Analysis Of A KPC-2-Producing Klebsiella Pneumoniae ST11 Outbreak From A Teaching Hospital In Shandong Province, China. Infect Drug Resist. 2019. 12: 29612969 .

[21] Wassef M, Abdelhaleim M, AbdulRahman E, Ghaith D. The Role of OmpK35, OmpK36 Porins, and Production of $\beta$-Lactamases on Imipenem Susceptibility in Klebsiella pneumoniae Clinical Isolates, Cairo, Egypt. Microb Drug Resist. 2015. 21(6): 577-80.

[22] Petrosillo N, Giannella M, Lewis R, Viale P. Treatment of carbapenem-resistant Klebsiella pneumoniae: the state of the art. Expert Rev Anti Infect Ther. 2013. 11(2): 159-77.

[23] Nwafia IN, Ohanu ME, Ebede SO, Ozumba UC. Molecular detection and antibiotic resistance pattern of extended-spectrum beta-lactamase producing Escherichia coli in a Tertiary Hospital in Enugu, Nigeria. Ann Clin Microbiol Antimicrob. 2019. 18(1): 41.

[24] van Hoek A, Veenman C, Florijn A, et al. Longitudinal study of ESBL Escherichia coli carriage on an organic broiler farm. J Antimicrob Chemother. 2018. 73(12): 3298-3304.

[25] Kazmierczak KM, de Jonge B, Stone GG, Sahm DF. Longitudinal analysis of ESBL and carbapenemase carriage among Enterobacteriaceae and Pseudomonas aeruginosa isolates collected in Europe as part of the International Network for Optimal Resistance Monitoring (INFORM) global surveillance programme, 2013-17. J Antimicrob Chemother. 2020. 75(5): 1165-1173. 
[26] Tewabe T, Fenta A, Tegen A, Mezgebu M, Fentie T, Zeleke T. Clinical Outcomes and Risk Factors of Meningitis among Children in Referral Hospital, Ethiopia, 2016: A Retrospective Chart Review. Ethiop J Health Sci. 2018. 28(5): 563-570.

[27] Karvouniaris M, Brotis AG, Tsiamalou P, Fountas KN. The Role of Intraventricular Antibiotics in the Treatment of Nosocomial Ventriculitis/Meningitis from Gram-Negative Pathogens: A Systematic Review and Meta-Analysis. World Neurosurg. 2018. 120: e637-e650.

[28] Chen Y, Li F, Zhu M, Liu L, Luo Y. Outcome and factors of patients with nosocomial meningitis by multi-drug-resistant Gram-negative bacteria in a tertiary hospital in China: a retrospective study. Br $\mathrm{J}$ Neurosurg. 2020 : 1-5.

[29] Olson D, Lamb MM, Gaensbauer JT, et al. Risk Factors for Death and Major Morbidity in Guatemalan Children with Acute Bacterial Meningitis. Pediatr Infect Dis J. 2015. 34(7): 724-8.

[30] Hanko M, Soršák J, Snopko P, Opšenák R, Zeleňák K, Kolarovszki B. Incidence and risk factors of early postoperative complications in patients after decompressive craniectomy: a 5-year experience. Eur J Trauma Emerg Surg. 2020 .

[31] McCormick DW, Wilson ML, Mankhambo L, et al. Risk factors for death and severe sequelae in Malawian children with bacterial meningitis, 1997-2010. Pediatr Infect Dis J. 2013. 32(2): e54-61.

[32] O'Grady NP, Alexander M, Burns LA, et al. Guidelines for the prevention of intravascular catheterrelated infections. Am J Infect Control. 2011. 39(4 Suppl 1): S1-34.

[33] Lu P, Raynald, Liu W, et al. Risk Factors of External Ventricular Drainage-Related Infections: A Retrospective Study of 147 Pediatric Post-tumor Resection Patients in a Single Center. Front Neurol. 2019. 10: 1243.

[34] Arabi Y, Memish ZA, Balkhy HH, et al. Ventriculostomy-associated infections: incidence and risk factors. Am J Infect Control. 2005. 33(3): 137-43.

[35] Eymann R, Chehab S, Strowitzki M, Steudel WI, Kiefer M. Clinical and economic consequences of antibiotic-impregnated cerebrospinal fluid shunt catheters. J Neurosurg Pediatr. 2008. 1(6): 444-50.

[36] Lyke KE, Obasanjo OO, Williams MA, O'Brien M, Chotani R, Perl TM. Ventriculitis complicating use of intraventricular catheters in adult neurosurgical patients. Clin Infect Dis. 2001. 33(12): 2028-33.

[37] Fu W, Shi N, Wan Y, et al. Risk Factors of Acute Gastrointestinal Failure in Critically Ill Patients With Traumatic Brain Injury. J Craniofac Surg. 2020. 31(2): e176-e179.

\section{Hosted file}

Table-0921.pdf available at https://authorea.com/users/380091/articles/496205-molecularepidemiology-and-survival-analysis-of-nosocomial-meningitis-induced-by-multi-drugresistance-enterobacteriaceae

\section{Hosted file}

Figure-0921.pdf available at https://authorea.com/users/380091/articles/496205-molecularepidemiology-and-survival-analysis-of-nosocomial-meningitis-induced-by-multi-drugresistance-enterobacteriaceae 